\title{
Introduction to the Wireless Networks minitrack
}

\author{
Edoardo Biagioni \\ University of Hawaii at Manoa \\ esb@hawaii.edu
}

\author{
John McEachen \\ Naval Postgraduate School \\ mceachen@nps.edu
}

\author{
Murali Tummala \\ Naval Postgraduate School \\ mtummala@nps.edu
}

\section{Wireless Networks}

Security and performance are two important properties of practical wireless networks. Without security, modern wireless networks are very limited in the benefits they may offer. This is particularly true of the public-access ("cellphone") networks that most of us use on a daily basis.

Modern wireless networks also require high performance - most of the motivation for transitioning from the current $4 \mathrm{G}$ to the future $5 \mathrm{G}$ is expected increases in performance, which can be used to provide enhanced applications such as high-quality video streaming. This performance must be provided at minimum cost, and in many applications, cost is the more important metric.

Both security and performance are notoriously difficult to achieve in practice. In the case of security, attackers might be more knowledgeable than defenders, have the initiative, and may trigger a range of harmful behavior to affect user communications. In a similar fashion, performance has a number of metrics, and optimizing for one often leads the other performance measures to be worse.

\section{Papers in this minitrack}

This minitrack has four papers, one on security and three on performance evaluation. The security paper identifies an LTE weakness that could lead to denialof-service for LTE devices, and proposes a remedy that might forestall such attacks.
The next paper uses data collected as part of a business - collecting tolls on German highways - to evaluate the latency and bandwidth of a large GSM 2G network. While 2G is largely obsolete as a technology for mobile telephone devices, it is still of interest as the low-cost solution for low-data rate devices such as have been envisioned for the Internet-of-Things (IoT).

The third paper is also related to the IoT, in that it studies the application of a specific technology, IEEE 802.11p, for communication between railway equipment such as train cars, and track-side equipment. For realistic vehicle speeds and equipment distances, the time for communication is very limited, so overall communication latency is the most important criterion. This paper compares the performance of 802.11p to 802.11 used in IBSS (ad-hoc) mode, and in BSS/AP (access point) mode.

The final paper focuses on the challenge of doing a realistic evaluation of wireless ad-hoc protocols. The author has built and deployed a 5-hop network using Raspberry Pi Zero-W embedded devices, and reports on the benefits and challenges of using them to evaluate and improve an existing wireless ad-hoc network.

\section{About this minitrack}

This minitrack was first held in 2005, and the track chairs are unchanged since 2011. As can be seen by the selection of this year's papers, we welcome submissions on any aspect of wireless networks technical, of course, but also social, economic, cultural, and others. 